\title{
Rhetorical Coding of Health Promotion Dialogues
}

\author{
Floriana Grasso \\ Department of Computer Science \\ University of Liverpool, UK \\ floriana@csc.liv.ac.uk
}

\begin{abstract}
Health promotion is a complex activity that requires both explanation and persuasion skills. This paper proposes a three-layered model of dialogue coding, based on a rhetorical argumentation model, and a behavioural model of change. The model was applied to the analysis of a corpus of 40 e-mail dialogue exchanges on healthy nutrition advice. Examples of analysis are given.
\end{abstract}

\section{Introduction}

Despite the benefits of a balanced diet are nowadays commonplace, research has shown that the promotion of healthy nutrition has to face stereotypes and prejudices $[1,2]$. It has become clear that mere informational and educational skills cannot be enough for these scenarios, for when people are not ready to accept advice, confrontation and argumentation are very likely to take place.

In our research, we seek to build advice giving systems that embed rhetorical argumentation skills, with the hope of providing more effective advice. In other papers, we have presented an architecture for an advice giving system based on rhetorical argumentation [3], and a variation of our conversational health promotion model, based on WWW interactions [4]. In the present paper, we are not concerned with computational issues, nor with the gained insights from the health promotion point of view, which are described in the above mentioned works. Instead, we want to focus this paper on the health promotion dialogues themselves, with the aim of capturing their fundamental peculiarities. A systematic analysis of dialogues, or in general human produced texts, is useful to build computational systems, both because it produces training material for systems developers, and because it provides benchmarks for the evaluation of these systems' output. We propose a three-layered coding scheme for the analysis of persuasive dialogues, which is grounded both on a behavioural model, and on the classical philosophy of argument. We apply the scheme to the analysis of a corpus of e-mail dialogues, which was collected to inform the above mentioned computational systems. Before entering into the details of the coding scheme, we spend the next section to briefly discuss these two theoretical grounds. 


\section{Health Promotion: Argumentative Models of Change}

The Stages of Change Model [5], a widely accepted theoretical model that explains how people modify their behaviour, suggests that individuals progress through very distinct stages of change on their way to change habits. From a first precontemplation stage, when people see no problem with their current behavior, a contemplation stage marks the moment when people come to understand their problem, and start thinking about solving it, though with no immediate plans. In a following preparation stage, people plan to take an action in the immediate future, and have already made some small changes in this direction. The action stage identifies people who are in the process of actively making behavior changes, until a maintenance stage is reached, where the new behavior is continued on a regular basis. In each of the stages, an advisor can use various strategies to foster movement to the next stage. These strategies are mainly information based for the more advanced stages of change, but it has become clear that the first passage, from the precontemplation to the contemplation stage, cannot be based only on the provision of new information [6]. It has been argued $[7,8]$ that more efficacy can be obtained, in these cases, by appealing to techniques coming from fields like classical argumentation.

The classical philosophy of argument is, not surprisingly, an attractive source of insights for researchers in dialogue processing. Many have been inspired by Toulmin's model of argument structure [9], but for a long time this model has been the almost exclusive point of contact between the two fields. Recently, however, a series of events has given the two communities the chance to meet $[10$, 11, e.g.], and works from new collaborations have started to ripe. We are among those who believe that the philosophy of argument has great potential for exploitation, and in particular we ground our work on the New Rhetoric (NR) [12], a seminal theory of argumentation. Dealing with discoursive techniques, the NR theory not only classifies what premises are appropriate in argumentation, but is especially concerned with how these premises are presented to the audience, for the exposition of the argument is sometimes more important than its validity. The NR is, in fact, a collection of ways, schemata, for arranging premises and conclusions that are successfully used by people in ordinary discourse. The main objective of a schema is to exploit associations among concepts, either known or new to the audience, in order to pass the audience's acceptance (positive or negative) from one concept to another. On the basis of this theory, we have developed a framework for rhetorical argumentation [13], which we have applied to the generation of health promotion dialogues [3].

\subsection{A Corpus of Argumentative Dialogues}

One thing is to take inspiration from theoretical works, well established as they might be. A completely different thing is, however, to verify whether the theoretical insights find confirmation in experimental practices. In order to pursue the latter objective, we conducted an experiment from which we obtained a corpus of "argumentative" dialogues, on the subject of healthy nutrition advice. The 
Table 1. Extracts from the corpus of e-mail dialogues

\section{Dialogue 1}

HP Do you like cooking?

A Not especially. [...] Cooking feels to me like a lot of effort for something (ie. eating) that's over quite quickly. Also, I often feel tired at the end of a day's work and don't want to spend too much time in the kitchen.

HP You do not cook just because you have to eat! Cooking can be a very relaxing and interesting activity, better than watching TV!

A I know you're right but that still doesn't make it easy to do!

\section{Dialogue 2}

HP Have you ever considered having some fruit for breakfast or as a snack?

B I should do that, yes. I'll have to go and buy some....

HP Don't you have to go and buy chocolate as well?

B I didn't mean it would take an extra effort to buy fruit on my trips to the supermarket. However [...] it's much easier to get hold of unhealthy snack food near work than it is to get fruit.

\section{Dialogue 3}

C I do enjoy fruit, but it is not as appealing as say a biccie, and vegetables in summer aren't really the go, I would eat vegies in the winter time at least 4 times a week.

HP Maybe pears and apples are not that appealing, but what about satsumas, or a cup of strawberries, or a bunch of grapes? Nice and refreshing, especially in summer!

C Yummy, I think if someone was to give me a plate of cut up fruit like below then I would definitely eat it, it is always more appealing when it is all done for you.

corpus consists of a collection of 46 e-mail dialogues, of varying length, with an average of 11 messages per dialogue, with the longest dialogue consisting of 45 exchanges. These were all two-party exchanges, where the investigator played the "health promotor" role, in a dialogue with a second party. These interlocutors were recruited from subscribers to a mailing list with interests in nutrition. Excerpts of some of these dialogues are shown in Table 1, where the health promotor's turns are labelled HP, and those of the different interlocutors' are labelled A, B, and C. The HP messages were generated semi-automatically, as the experiment was conducted as an evaluation of a computational argumentation system, as described in [3]. The generation was based on a preliminary study in which the investigators played the opposite role of "advisees" in dialogues with real nutritionists.

\section{A Three-Layered Coding Scheme}

It has been recognised by many (see, f.i., [14]) that the Speech Act theory [15] hypothesis that each utterance can be associated one single goal is not satisfactory, as the same speech act can serve to many purposes. A dialogue coding scheme should therefore have a more complex view on how the two partners 
contribute to the dialogue, in terms of their hierarchy of goals. We propose a three-layered structure to represent dialogues, where each utterance, or dialogue move, can be seen from three perspectives:

1. a meta-goal level, that is the ultimate goal of the dialogue for a partner, the reason why the dialogue has been initiated;

2. the rhetorical level, that is what kind of rhetorical goals/strategies a portion of the dialogue shows;

3. the move level, that is the dialogue moves that have been actually used to convey the above goals.

We better elaborate on the three levels in what follows.

\subsection{Meta-dialogue Moves}

Meta-dialogue moves identify the dialogue higher order goals. Typically there will be one high order goal per dialogue, although this is not prescribing. In our case, these goals are characterised by specific strategies associated with the Stages of Change model. We want to explicitly mark the following meta-moves:

- From the advisor agent point of view:

1. exploratory moves: the portion of dialogue turns used to establish in which stage of change the advisee is;

2. action moves: the portion of the dialogue in which the advisor applies one of the strategies to encourage the advisee to move a stage further;

- From the advisee agent point of view:

3. declarative moves: the portion of the dialogue in which the advisee gives information on the stage he is in;

4. responsive moves: the portion of the dialogue in which the advisee accepts, or shows resistance to, the state change;

Information useful to characterise the above moves, is, in our case, grounded on various literature on behavioural research [16, for example].

The meta-moves are designed to apply to general argumentative dialogues, not necessarily on health promotion, where the "stage of change" can be substituted by any "position" the "opponent" might be in, and the strategies can vary from domain to domain. In a general situation, also, the dialogue is not necessarily asymmetric: the two participants may both have a meta-goal to achieve, and strategies to pursue it. Therefore, both participants can in turn be in any of the two roles ("attack" and "defend") and play each of the four meta-moves, according to the situation.

\subsection{Rhetorical Moves}

By rhetorical moves we intend the moves which are specifically used for the argumentation, according to specific classical argumentation techniques, and in order to satisfy the higher level goal, or goals. For defining these moves, we 
base ourselves, as we anticipated, on the New Rhetoric model of argumentation. The theory lists $30+$ techniques, or schemata, of argumentation, with examples of their application. In [13] we give a formalisation of each of these techniques in the form of a schema. The schema not only identifies the way in which the techniques is applied, but also defines a series of applicability constraints that will make the argument stronger, or effective. We define a schema as a 6-tuple $R_{S}=\left\langle N, C, O_{c}, A_{c}, R_{c}, S_{c}\right\rangle$ where:

$-N$ is the name of the schema,

$-C$ is the claim the schema supports,

- $O_{c}$ are the ontological constraints the schema is based on, that is which relations should exist in the domain ontology among the concepts used in the schema for the schema to be applicable;

- $A_{c}$ are the acceptability constraints, that is which beliefs the party the argument is addressed to has to possess for the schema to be effective;

$-R_{c}$ are the relevance constraints, that is which beliefs the party the argument is addressed to has to have "in focus", that is which beliefs are relevant to the argument;

- $S_{c}$ are the sufficiency constraints, that is which premises should exist in order for the argument not to be weak, or attackable. This is perhaps the most elusive of the constraints, and it will vary from schema to schema.

An appropriate application of the pragmatic schema in a dialogue would assume that the arguer has either supposed, or has verified, all the constraints. For instance, the pragmatic argument is one of the techniques proposed in the NR which aims at evaluating an act or an event in terms of its positive or negative consequences, either observed, foreseen or even purely hypothetical. An example of pragmatic argument in our scenario could be: since eating apples helps slimming, and being slim is important to you, then you should eat apples. The instantiated definition of the pragmatic schema will be:

Claim: "Apples should be eaten";

Ontological constraints: "Apples are edible"; "Apples help sliming";

Acceptability constraints: "the addressee believes being slim is a good thing"; Relevance constraints: "the addressee is aware, or should be made aware, that apples help slimming". If this already holds, the argument can be put forward in a short form you should eat apples for you would like to slim.

Sufficiency constraints: an effort should be made to show that the action is necessary and sufficient for the consequence to happen, for instance by supporting statistics. Also, it might be shown that eating apples has no negative consequences, especially from a perspective similar to the one is being made (e.g. if eating apples helps slimming but, say, causes skin redness, this would not be acceptable to a person who is concerned about look).

\subsection{Dialogue Moves}

At the lowest level, the dialogue structure comprises single dialogue moves, typically extracted from a pre-established set of basic moves. There is debate as 
to how many and which types of moves should be needed, but we are for a parsimonious approach, in the spirit that the effort of distinguishing between moves of different types is only justified by a correspondent relief in the effort of understanding the intention of the move. We distinguish among four main move types, some of them comprising a number of subtypes:

1. Assertions: including all general assertive utterances an agent can perform, that is all the moves in which the agent makes a point. An assertion can be one of:

(a) Claims, that is statements which, although might address previous point in the conversation, are put forward with the aim of making a new point.

(b) Backings, that is statements that support one's own claims.

(c) Acknowledgements, that is statements which agree/accept another agent's point, or "re-state" one own's point.

(d) Replies, that is statements which reply to questions previously posed.

(e) Disputations, that is statements which explicitly disagree on previously made points.

(f) Withdrawals, that is statements which explicitly deny one's own previously made points.

2. Queries: including one agent's queries to which the interlocutor is supposed to reply with an assertion. These comprise:

(a) Open Questions: these are requests for information, on items the querying agent does not suppose previous knowledge. Note that the open questions do not refer to any previous move in the dialogue.

(b) Requests for Argument: these are requests for an argument in support of the claim expressed in the content of the move. Again, this request is made with respect to one generic claim, and does not refer to any previous move in the dialogue, as opposed to what happens with the:

(c) Challenges of Claim: these are requests to provide an argument supporting a claim previously made by another agent.

3. YN queries: we have included in a class of their own closed questions, that is questions whose only reply can be either Yes (True) or No (False).

4. YN: similarly, a separate class identifies answers to a YN-question.

\section{Analysing Argumentative Dialogues}

The annotation of a dialogue according to a code scheme is aimed at reconstructing the structure of the dialogue, and of the participant agents' goals. Our three-layered analysis can be done on a form as shown in Table. 2. The middle part of the form (Dialogue) lists the numbered dialogue moves, as broken down by the annotator into "basic unit". A basic unit can be an entire speaker's turn, or a sentence in a turn, or smaller parts of a sentence, according to the analyst's judgement. The moves of the two agents are annotated separately, in the left and right sections of the table.

Assuming a bottom-up approach to the analysis, starting from the move level, up to the meta-level, the analyst will annotate, in the two columns headed 
Move, the specific dialogue move used, according to the list in Sect. 3.3. For each move, the move type is indicated, as well as, if appropriate, the number of referenced preceding move. In a second phase, the analyst will look for occurrences of rhetorical schemata. Instances of the speaker assuming, or specifically testing, or actively meeting the constraints of a schema, as explained in Sect. 3.2, are identified, as well as the putting forward of the schema's claim. The two columns headed Schema will be filled in at this stage, with the specific mention of the constraint satisfaction process. Note that a schema can span over several dialogue turns. The final, in a bottom-up approach, phase looks for manifestations of the two agents' higher order moves, as explained in Sect. 3.1. In our health promotion dialogues, the meta-moves will be labelled with the stages of change that is currently being acted upon.

Table 2 shows an example of annotation, applied to Dialogue 1 of Table 1. For the sake of brevity, the example does not show how the application of the argumentative schema has been checked against the constraints. In Move 1, a Yes-No question, Agent 1 makes an Exploratory meta-move to establish the stage of change of the other agent with respect to the habit of preparing one's food, rather than buying pre-packed meals. Agent 2 makes an extensive Declarative meta-move, by replying to the question (Move 2) and providing extra information in support. Move 3 is a Backing for the dislike of cooking, which is done with a schema of argument by Ends and Means: the end (quick eating) does not justify the effort to put in the means (cooking). Another argument is supplied, in the Claim of Move 4, which appeals to the Motives behind the behaviour: time to relax is precious and should not be wasted cooking. Agent 1 triggers the Action meta-goal and starts providing an argument with the Claim in Move 5, and its Backing in Move 6. The argumentative technique used is Dissociation, in order to change the opponent's perspective: this breaks the link between cooking and eating, and replaces it with a link between cooking and relaxing, something that the opponent has just claimed to value. Agent 2 adopts a Responsive meta-goal, which shows that the stage change is not yet accepted. An argument "From the Easy" is used, which is meant to value that which is easy and possible, versus that which is difficult. The Acknowledgement in Move 7 serves again to change the perspective under consideration: the "interestingness" of the activity is recognised, but it is valued less than its unpleasantness, or difficulty to do.

\section{Related Research}

The use of rhetorical notions to annotate text goes back to the seminal work of Mann and Thompson, in their "Rhetorical Structure Theory" (RST) [17]. Although not specifically designed for dialogues, the theory has had various elaborations and variations to make it applicable to a great variety of texts, including dialogues. However, the theory has strong shortcomings in the representation of argumentation texts [18]. An example of application of RST to dialogue coding is the one in [19], where argumentative spoken dialogues are annotated. However, no higher level goal are considered, and the "argument schemas" are in fact 
Table 2. An annotated dialogue fragment

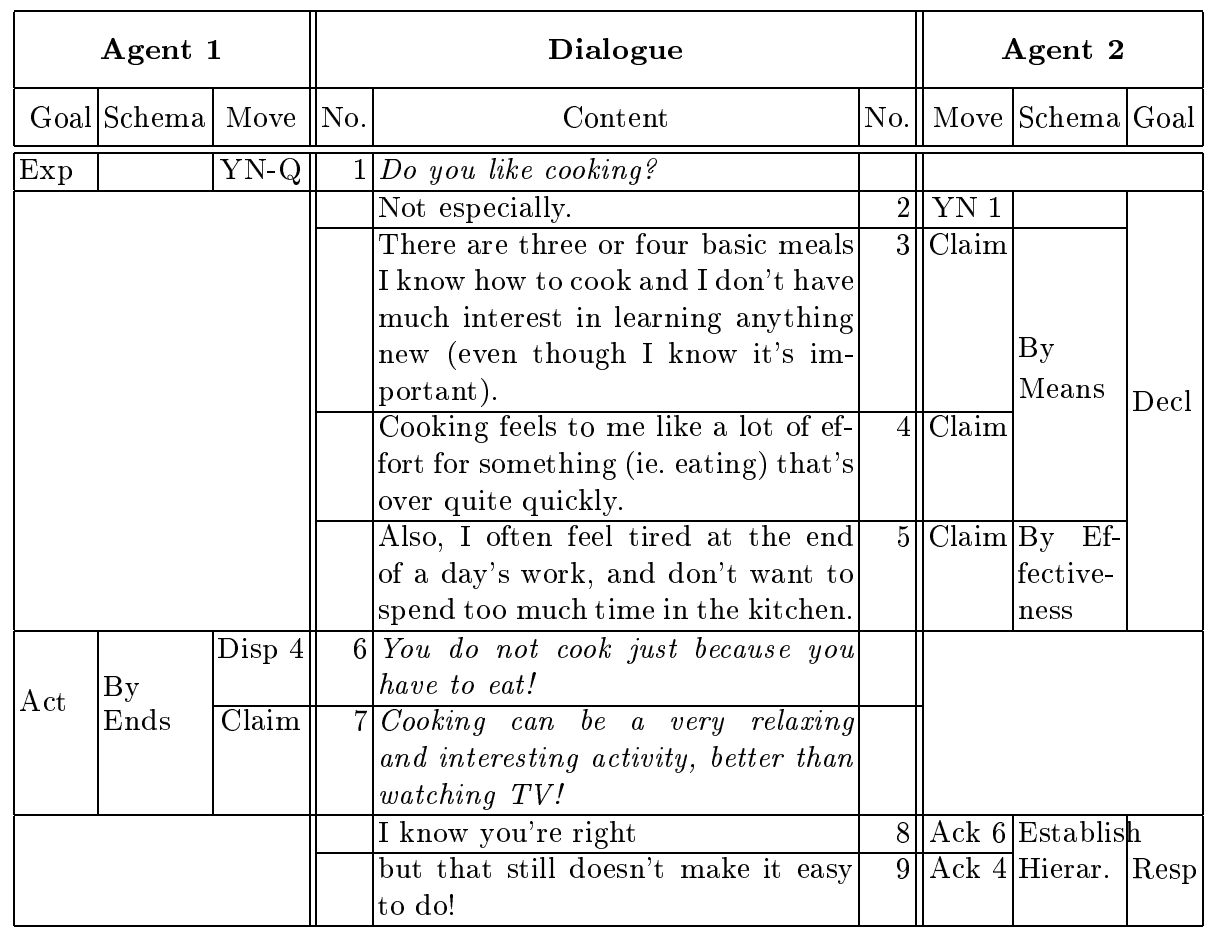

the basic RST constructs, which, as mentioned before, do have various problems with argumentative discourse. In [20] an annotation theory is proposed for argumentative texts. The authors concentrate on research papers, of which the argumentative nature is captured of sections like the description of background research, or the comparison with other works. They address, therefore, a monological situation, rather than a dialogical one. An important dialogue coding scheme is the one described in [21], which identifies, like ours, three levels of dialogue structure: (i) conversational moves are the basic units of the dialogue structure. (ii) Conversational games are sequences of moves fulfilling a specific goal; games can be embedded (for instance for clarification subdialogues), and are typically identified with the name of the first move of the game. Finally, (iii) transactions are sequences of games which satisfy a given, higher level purpose. The levels, however, do not account for specific rhetorical strategies, nor for how one level serves to the purposes of its predecessor. Another example of multi-layered analysis is the one in [22]. In this work, three classes of moves are defined, that are meant to identify the phenomena of (i) forward direction, that is sentences which are are said in order for something to happen, (ii) backward direction, that is sentences that are directed to the past of the dialogue, e.g. acknowledgements or agreements, (iii) and form and content of the utterances. The work does not however concentrate on dialogue meta-goals, nor, once again, captures the rhetorical organisation of the dialogue. 
Perhaps the best known example of the use of argumentation techniques in medical environments is due to Fox \& Das [23]. The work presented in this paper, conversely, is not concerned with argumentative reasoning, but focuses on the analysis of natural occurring argumentative dialogues in health promotion environments.

\section{Conclusions and Further Works}

We have presented a coding scheme to annotate argumentative dialogues in the domain of health promotion. Our approach, to the best our knowledge, is the only one which espouse the need for precisely capture the argumentative nature of the dialogues, by appealing to a classical theory from the philosophy of argument, with the way in which the argument serves to higher level goals of the participants, by appealing to a well established behavioural model.

An important test for any annotation scheme is its reliability, that is the fact that it can be applied and used by people other than the developers, and that the same analysts will give similar analyses over time [24]. This is the single most important step that is still in progress: so far the analyses have been done by the developers, although sometimes by consultations with colleagues. A trial study is at the moment being conducted, with a set of annotators, trained on the coding scheme, but not necessarily familiar with either the domain or the theories behind the scheme. The complete set of statistics from the study is not available at the time of writing, but informal considerations seem encouraging in supporting the assumption that indeed the three layers do capture the behaviour of argumentative dialogues.

\section{References}

1. Fries, E., Croyle, R.: Stereotypes Associated with a Low-Fat Diet and their Relevance to Nutrition Education. Journal of the American Dietetic Association 93 (1993) 551-555

2. Sadalla, E., Burroughs, J.: Profiles in Eating: Sexy Vegetarians and Other DietBased Social Stereotypes. Psychology Today 15 (1981) 51-57

3. Grasso, F., Cawsey, A., Jones, R.: Dialectical Argumentation to Solve Conflicts in Advice Giving: a case study in the promotion of healthy nutrition. International Journal of Human-Computer Studies 53 (2000) 1077-1115

4. Cawsey, A., Grasso, F., Jones, R.: A Conversational Model for Health Promotion on the World Wide Web. [25] 379-388

5. Prochaska, J., Clemente, C.D.: Stages of Change in the Modification of Problem Behavior. In Hersen, M., Eisler, R., Miller, P., eds.: Progress in Behavior Modification. Volume 28. Sycamore Publishing Company, Sycamore, IL (1992)

6. Prochaska, J.: Strong and Weak Principles for Progressing from Precontemplation to Action on the Basis of Twelve Problem Behaviors. Health Psychology 13 (1994)

7. Cawsey, A., Grasso, F.: Goals and Attitude Change in Generation: a Case Study in Health Education. In Jokinen, K., Maybury, M., Zock, M., Zukerman, I., eds.: Proceedings of the ECAI-96 Workshop on: Gaps and Bridges: New directions in Planning and NLG. (1996) 19-23 
8. Reiter, E., Robertson, R., Osman, L.: Types of Knowledge Required to Personalize Smoking Cessation Letters. [25] 389-399

9. Toulmin, S.: The Uses of Argument. Cambridge University Press (1958)

10. Reed, C., Norman, T., eds.: Symposium on Argument and Computation: position papers. In Reed, C., Norman, T., eds.: Symposium on Argument and Computation: position papers, http://www.csd.abdn.ac.uk/ ${ }^{\text {tnorman/sac/ (2000) }}$

11. Carenini, G., Grasso, F., Reed, C., eds.: Proceedings of the ECAI 2002 workshop on Computational Models of Natural Argument. In Carenini, G., Grasso, F., Reed, C., eds.: Proceedings of the ECAI 2002 workshop on Computational Models of Natural Argument. (2002)

12. Perelman, C., Olbrechts-Tyteca, L.: The New Rhetoric: a treatise on argumentation. University of Notre Dame Press, Notre Dame, Indiana (1969)

13. Grasso, F.: Towards a framework for rhetorical argumentation. In Bos, J., Foster, M., Matheson, C., eds.: EDILOG'02: Proceedings of the 6th workshop on the semantics and pragmatics of dialogue, Edinburgh (2002) 53-60

14. Cohen, P., Levesque, H.: Rational Interaction as the Basis for Communication. In Cohen, P., Morgan, J., Pollack, M., eds.: Intentions in Communication. MIT Press, Cambridge, MA (1990) 221-255

15. Searle, J.: Speech Acts: An essay in the philosophy of language. Cambridge University Press, Cambridge (1969)

16. Barrie, K.: Motivational Counselling in Groups. In Davidson, R., Stephem, R., MacEwan, I., eds.: Counselling Problem Drinkers. Tavistock/Routledge, London (1991)

17. Mann, W., Thompson, S.: Rhetorical Structure Theory: Toward a Functional Theory of Text Organization. Text 8 (1988) 243-281

18. Reed, C., Long, D.: Generating the structure of argument. In: Proceedings of the 17th International Conference on Computational Linguistics and 36th Annual Meeting of the Association for Computational Linguistics (COLING-ACL'98). (1998) 1091-1097

19. Stent, A., Allen, J.: Annotating Argumentation Acts in Spoken Dialog. Technical Report 740, The University of Rochester, Computer Science Department (2000) (TRAINS Technical Note 00-1).

20. Teufel, S., Carletta, J., Moens, M.: An Annotation Scheme for Discourse-Level Argumentation in Research Articles. In: Proceedings of EACL. (1999)

21. Carletta, J., Isard, A., Isard, S., Kowtko, J., Doherty Sneddon, G., Anderson, A.: The Reliability of a Dialogue Structure Coding Scheme. Computational Linguistics 23 (1997) 13-31

22. Core, M., Allen, J.: Coding dialogs with the DAMSL annotation scheme. In Traum, D., ed.: AAAI Fall Symposium on Communicative Action in Humans and Machines. (1997)

23. Fox, J., Das, S.: Safe and Sound: Artificial Intelligence in Hazardous Applications. AAAI Press / The MIT Press (2000)

24. Carletta, J.: Assessing Agreement on Classification Tasks: the kappa Statistic. Computational Linguistics 22 (1996) 249-254

25. P.W.Horn, Shahar, Y., Lindberg, G., S.Andreassen, Wyatt, J., eds.: Artificial Intelligence in Medicine. Proceedings of the Joint European Conference on Artificial Intelligence in Medicine and Medical Decision Making, AIMDM'99. Volume 1620 of LNAI., Springer-Verlag (1999) 